Revista Calidad en la Educación Superior

Programa de Autoevaluación Académica

Universidad Estatal a Distancia, Costa Rica

ISSN 1659-4703

revistacalidad@uned.ac.cr

\title{
ESTRATEGIA EVALUATIVA EN MIRAS DEL APRENDIZAJE AUTÓNOMO Y CREATIVO DE LA PERSONA ESTUDIANTE: CASO CURSO DE TURISMO SOSTENIBLE UNIVERSIDAD ESTATAL A DISTANCIA, COSTA RICA
}

\author{
EVALUATIVE STRATEGY IN VIEW OF SELF AND CREATIVE PERSON STUDENT \\ LEARNING: CASE COURSE IN SUSTAINABLE TOURISM STATE DISTANCE \\ UNIVERSITY, COSTA RICA
}

\author{
Susan Solís Rosales ${ }^{1}$ \\ ssolis@uned.ac.cr \\ Universidad Estatal a Distancia, Costa Rica
}

\author{
Volumen 7, Número 1 \\ Mayo 2016 \\ pp. $321-337$
}

Recibido: 23 de octubre, 2015

Aprobado: 07 de marzo, 2016

\footnotetext{
1 Magister Scientiae en Desarrollo Económico Local con énfasis en Turismo Comunitario de la Facultad Latinoamericana de Ciencias Sociales (FLACSO-CR) y el Instituto Tecnológico de Costa Rica (TEC). Actualmente, directora de la Cátedra Turismo Sostenible de la Escuela de Ciencias Sociales y Humanidades de la UNED-CR. Correo electrónico: ssolis@uned.ac.cr.

Ponencia presentada en el primer Congreso Internacional de Innovación Educativa del Instituto Tecnológico de Monterrey, Ciudad de México.
} 


\title{
Resumen
}

Para el uso efecto de las tecnologías de información y de comunicación el ser humano requiere desarrollar las siguientes habilidades: creatividad, autorregulación y autoevaluación. Este es un gran reto de los docentes; no obstante, se tiene una excelente oportunidad en utilizar la evaluación de los aprendizajes como estrategia de aprendizaje para la persona estudiante y el docente.

Se requiere cambiar de paradigma, en donde se ha pensado o se piensa dejar la evaluación de los aprendizajes solo para la medición del conocimiento (usualmente el nivel de memoria) que está obteniendo el estudiante. Dicha evaluación es importante y un recurso más de evidencia; sin embargo, el contexto nos demanda ser más propositivos desde la evaluación alternativa.

En el caso particular, bajo la metodología de estudio de caso se expone la experiencia de la evaluación alternativa aplicada a un curso de turismo sostenible, mediante las tecnologías de información y comunicación (TIC), aplicada en un modelo pedagógico a distancia, en el nivel de educación superior público en Costa Rica. Una práctica que está en proceso de construcción.

Palabras clave: evaluar para aprender, aprendizaje autónomo, evaluación alternativa, aprendizaje creativo, educación a distancia.

\begin{abstract}
Information technology and communication require that human beings develop the following skills: creativity, self-regulation and self-assessment. This is a gigantic challenge for teachers; however, the assessment of learning becomes an excellent opportunity since it can be employed as a learning strategy for the student and faculty.

A paradigm shift is required: stop thinking about the assessment of learning exclusively for the measurement of the student's "knowledge" (usually understood as the ability of memorizing). Such evaluation is important and a resource of evidence; however, the context demands more proactivity and constructive towards the alternative assessment.

In this particular case, the methodology of case study has been selected to analyze the experience of alternative assessment applied to a course of sustainable tourism. It is applied through information and communications technology (ICT) at a distance learning environment in a public higher education in Costa Rica. A practice that is under construction.
\end{abstract}

Keywords: Evaluate for learning, autonomous learning, alternative assessment, creative learning, distance education. 


\section{Introducción}

El tema de la evaluación de los aprendizajes es álgido por las diversas variables que se conjugan. Además, al parecer en la práctica ya estandarizada, resulta más cómodo para las instituciones aplicar las pruebas escritas, más que asumir el reto de cambiar la visión de una evaluación sumativa a una evaluación formativa (al menos de forma balanceada). Según el Programa de Apoyo curricular y evaluación de los aprendizajes (PACE) mencionando a Jorba y Casellas (1997, p.83) indican que la evaluación formativa es:

(...) el tipo de evaluación que se lleva a cabo durante todo el proceso de aprendizaje, la cual posee una función reguladora del proceso de enseñanza aprendizaje a fin de posibilitar que los medios de formación responda a las características del estudiantado. Tiende más a identificar cuáles son las deficiencias del aprendizaje que a determinar los resultados alcanzados con este aprendizaje.

Esa propuesta de evaluación formativa requiere más planeación, recursos, capacitación y, en el nivel macro, se esperaría un soporte de las políticas institucionales. Desde esta óptica, se podría mirar un gran coste; sin embargo del otro lado de la balanza, se tiene los beneficios que la persona estudiante adquiere. Por ejemplo: su inserción en los diversos contextos que la dinámica social le exija, el desarrollo autónomo de su aprendizaje, la creatividad, entre otros.

Y por parte de la institución, iniciaría su proceso en el cambio de paradigma de la evaluación estandarizada (sigue el mismo patrón de evaluación para medir resultados), por el paso paulatino de la evaluación alternativa que aprovecha los recursos que dispone la TIC (Tecnología de información y comunicación).

El presente caso comparte la experiencia práctica que se encuentra en proceso de construcción colectiva (a lo interno del cuerpo docente a cargo de los cursos de la cátedra turismo sostenible) ante las interrogantes: ¿cómo aplicar la TIC en la 
evaluación de los aprendizajes?, ¿cómo establecer una estrategia de aprendizaje mediante su evaluación?, ¿cómo la persona estudiante puede ser actor dinámico del proceso de aprendizaje?

Este es uno de los primeros pasos; a saber: cambiar en la práctica docente para la transformación.

\section{Aproximación conceptual}

La evaluación de los aprendizajes, según Barberà (2004) así como Bautista, Borges y Forès (2008) debe propiciar, en los diversos actores involucrados del proceso evaluativo (docente y estudiante), información para observar los niveles de avance en el aprendizaje que se está desarrollando y, a partir de aquí, aplicar acciones de mejora, si fuera el caso.

Al respecto, Álvarez (2001) menciona:

La evaluación actúa entonces al servicio del conocimiento y del aprendizaje, y al servicio de los intereses formativos a los que esencialmente debe servir. Aprendemos de la evaluación cuando la convertimos en actividad de conocimiento, y en acto de aprendizaje el momento de la corrección. Sólo cuando aseguramos el aprendizaje podremos asegurar la evaluación, la buena evaluación que forma, convertida ella misma en medio de aprendizaje y en expresión de saberes (p.2)

Por lo tanto, se puede definir la evaluación de los aprendizajes como la acción o el proceso que permite establecer la estrategia pedagógica, la cual, mediante la utilización de técnicas e instrumentos, generará la información y los datos en aras de retroalimentar en dos vías: quién aplica esa evaluación y quién es evaluado.

El equipo docente busca una evaluación que permita el aprendizaje y que este sea para la vida, es decir, que no quede relegado o suscrito al lapso de corto tiempo en una prueba escrita u otro tipo de instrumento que busque solamente certificar o 
medir un conocimiento. Es necesario promover uno de los principios básicos de educación para la vida: aprender a aprender. Al respecto, Calderón (2008) indica al no aplicar lo aprendido o propiciar su práctica en el recinto educativo, sea cual sea éste, carece de valor, no es significativo, es vacío. Así estamos aprendiendo, en el vacío. En el mejor de los casos tenemos nociones, pero no estamos propiciando ni promoviendo su práctica significativa (p.118)

En el caso particular de la Universidad Estatal a Distancia de Costa Rica (UNED), su modelo pedagógico tiene como eje central al estudiante, por ende, su enmienda es generar procesos de aprender a aprender y la autorregulación (Carmiol, 2010). Los aspectos anteriores son grandes retos en un contexto a distancia por la complejidad en su práctica; no obstante, son fundamentales para el desenvolvimiento de la persona estudiante. Una forma de alcanzar esos objetivos será en la reflexión y en el análisis de la estrategia evaluativa. Según Barberá (2004) refiriéndose a los resultados que la evaluación de los aprendizajes puedan suscitarse ante el estudiante:

Su autoconcepto como aprendiz será decisivo para afrontar con éxito las tareas educativas, en general, y no sólo durante su vida escolar, sino a lo largo de toda su existencia. Por lo tanto, el tratamiento de este hecho no es un tema banal, sino algo en lo que debemos volcar todo esfuerzo docente. Es necesaria una reflexión profunda sobre las prácticas evaluativas, puesto que unos métodos didácticos excelentes llevados a cabo impecablemente por esforzados profesores se ven traicionados por las alternativas elegidas en el momento de evaluar (p.40)

En contraste con lo expuesto Batista, Borges y Forès (2008) anteriormente, el ensayo y error en el cambio de las estrategias evaluativas que creen ambientes reflexivos e incentive al estudiante a la autonomía e implicación personal en su estudio, es una tarea de constante mejora que vuelve la acción de evaluar muy dinámica y estratégica, para optimizar el aprendizaje. En consecuencia, permitiría, 
con mayor posibilidad, la inserción de la persona estudiante en su futuro ámbito laboral, así como su formación como persona integral.

\section{Estrategia evaluativa en miras del aprendizaje autónomo y creativo del educando.}

\section{Contexto de la experiencia}

La UNED se creó en el año 1977, con el objetivo de democratizar la educación a las poblaciones que, por barreras de ubicación geográfica, económicas o laborales, se les dificultaba estudiar en educación superior (UNED, 2005). Para ello, la UNED tendría el mandato de generar procesos de enseñanza y aprendizaje, utilizando los diversos medios de comunicación existentes según contextos.

Actualmente, la experiencia que se presenta surge en un contexto en el cual se elaboró el Plan académico (2012-2017). Entre sus particularidades, se diseñó de forma participativa (convocó a representantes de la comunidad universitaria) y estableció un reto, así como líneas en el desarrollo de la evaluación para el aprendizaje (UNED, 2013).

La Cátedra Turismo Sostenible se ubica en la carrera de Gestión Turística Sostenible (en la Escuela de Ciencias Sociales y Humanidades) y tiene a cargo la asignatura Fundamentos de Gastronomía Costarricense (nivel de diplomado), la cual se ofertó en el III cuatrimestre (del 8 de setiembre al 14 de diciembre del 2014). Esta reportó una matrícula de 95 estudiantes; de los cuales. 50,5\% se ubican, geográficamente en el área metropolitana del país y el 49,5\% fuera del área metropolitana. Además, en cuanto al sexo, $70,5 \%$ son mujeres y el $29,5 \%$ son hombres. La modalidad del curso es a distancia, es decir, a la persona estudiante se le entregan los materiales didácticos y, a través de los diversos medios de comunicación (por ejemplo plataforma Moodle), dispuestos para su comunicación con el cuerpo docente, intercambia sus dudas y participa en actividades, así como la aplicación de instrumentos evaluativos, según fechas preestablecidas. 
Estrategia evaluativa en miras del aprendizaje autónomo y creativo de la persona estudiante: caso curso de turismo sostenible UNED-Costa Rica.

Susan Solís Rosales

Propuesta de la estrategia evaluativa

El objetivo de aprendizaje del curso es: Solís (2014)

brindar conocimientos de la cocina costarricense desde el punto de vista histórico y cultural, analizados desde dos perspectivas: gastronómica, con la evolución de las costumbres de mesa y todo lo que se relaciona con ello, y culinaria, que aporta técnicas de preparación de alimentos y todos los símbolos que éstas reflejan, lo que permitirá conocer y situar un aspecto básico de la cultura de nuestro país, en un entorno universal, incorporándola al desarrollo de la actividad turística de acuerdo con el desarrollo rural comunitario y de acuerdo con la sostenibilidad ambiental (p.2).

A continuación, se presentará los materiales y apoyos didácticos del curso en cuestión:

Tabla 1: Materiales y apoyos didácticos de la asignatura en estudio.

\begin{tabular}{|c|c|c|c|}
\hline Impreso & $\begin{array}{c}\text { Video } \\
\text { conferencia } \\
\text { (tutorías) }\end{array}$ & $\begin{array}{l}\text { En línea } \\
\text { (virtual) }\end{array}$ & $\begin{array}{l}\text { Programa de } \\
\text { Radio en línea } \\
\text { (REA) }\end{array}$ \\
\hline $\begin{array}{l}\text { Libro de texto } \\
\text { (desarrolla los } \\
\text { contenidos } \\
\text { temáticos) }\end{array}$ & $\begin{array}{l}\text { Espacio de } \\
\text { intercambio } \\
\text { entre docentes y } \\
\text { estudiantes. }\end{array}$ & $\begin{array}{l}\text { Apoyo en la } \\
\text { plataforma } \\
\text { Moodle } \\
\text { (espacio } \\
\text { complementario } \\
\text { de encuentro } \\
\text { entre docentes } \\
\text { y estudiantes) }\end{array}$ & $\begin{array}{l}\text { Como } \\
\text { complemento al } \\
\text { estudio de los } \\
\text { contenidos } \\
\text { temáticos, se } \\
\text { desarrolla tres } \\
\text { programas de } \\
\text { radio. } \\
\text { La influencia de la } \\
\text { cocina francesa en } \\
\text { Costa Rica } \\
\text { http://www.ondaun } \\
\text { ed.com/programa.p } \\
\text { hp?ou=206 }\end{array}$ \\
\hline $\begin{array}{l}\text { Guía de estudio } \\
\text { (acompaña en el } \\
\text { estudio al } \\
\text { estudiante) }\end{array}$ & $\begin{array}{l}\text { Se deja la } \\
\text { videoconferencia } \\
\text { grabada, para } \\
\text { que los } \\
\text { estudiantes } \\
\text { puedan } \\
\text { accederla en } \\
\end{array}$ & $\begin{array}{l}\text { Programa de } \\
\text { Apoyo didáctico } \\
\text { (PADD } \\
\text { La persona } \\
\text { estudiante } \\
\text { puede enviar } \\
\text { sus consultas a }\end{array}$ & $\begin{array}{l}\text { La tradición } \\
\text { culinaria de la } \\
\text { cultura china en } \\
\text { Costa Rica } \\
\text { http://www.ondaun } \\
\text { ed.com/programa.p } \\
\text { hp?ou=211 }\end{array}$ \\
\hline
\end{tabular}




\begin{tabular}{|c|c|c|c|}
\hline & $\begin{array}{l}\text { otro momento } \\
\text { http://videoteca } \\
\text { vau.uned.ac.cr/vi } \\
\text { deos/video/244/i } \\
\text { n/channel/3/ } \\
\text { (utilización de } \\
\text { recurso } \\
\text { educativo } \\
\text { abierto REA) }\end{array}$ & $\begin{array}{l}\text { través del fax, } \\
\text { teléfono o } \\
\text { correo } \\
\text { electrónico. }\end{array}$ & \\
\hline $\begin{array}{l}\text { Orientación } \\
\text { académica (traza } \\
\text { la ruta de } \\
\text { aprendizaje: } \\
\text { indicaciones } \\
\text { específicas del } \\
\text { curso, aspectos de } \\
\text { evaluación, } \\
\text { objetivos de } \\
\text { aprendizaje y lista } \\
\text { de cotejo) } \\
\text { http://sanpedro.une } \\
\text { d.ac.cr/orientacion } \\
\text { es/2014505155.pdf }\end{array}$ & & $\begin{array}{l}\text { Facebook } \\
\text { (REA) } \\
\text { https://es- } \\
\text { es.facebook.co } \\
\text { m/pages/C\%C3 } \\
\% \text { A1tedra-de- } \\
\text { Turismo- } \\
\text { Sostenible/1389 } \\
\text { 665407988520 }\end{array}$ & $\begin{array}{l}\text { La cocina } \\
\text { costarricense } \\
\text { desde los ojos de } \\
\text { un francés } \\
\text { http://www.ondaun } \\
\text { ed.com/programa.p } \\
\underline{\text { hp?ou=293 }}\end{array}$ \\
\hline
\end{tabular}

Fuente: Elaboración propia a partir del documento orientación académica (Solís 2014).

En la tabla 1, se evidencia los diversos medios de comunicación utilizados para generar un acercamiento con el contenido temático y la persona estudiante con el acompañamiento docente respectivo. Estas serán las diferentes vías por las cuales el contenido se expondrá, a partir de las posibilidades de acceso geográfico, virtual, de tiempo y estilo de aprendizaje de la persona estudiante.

A continuación, en la figura 1 se desglosará la estrategia evaluativa utilizada: 
Figura 1: Propuesta estrategia evaluativa del curso

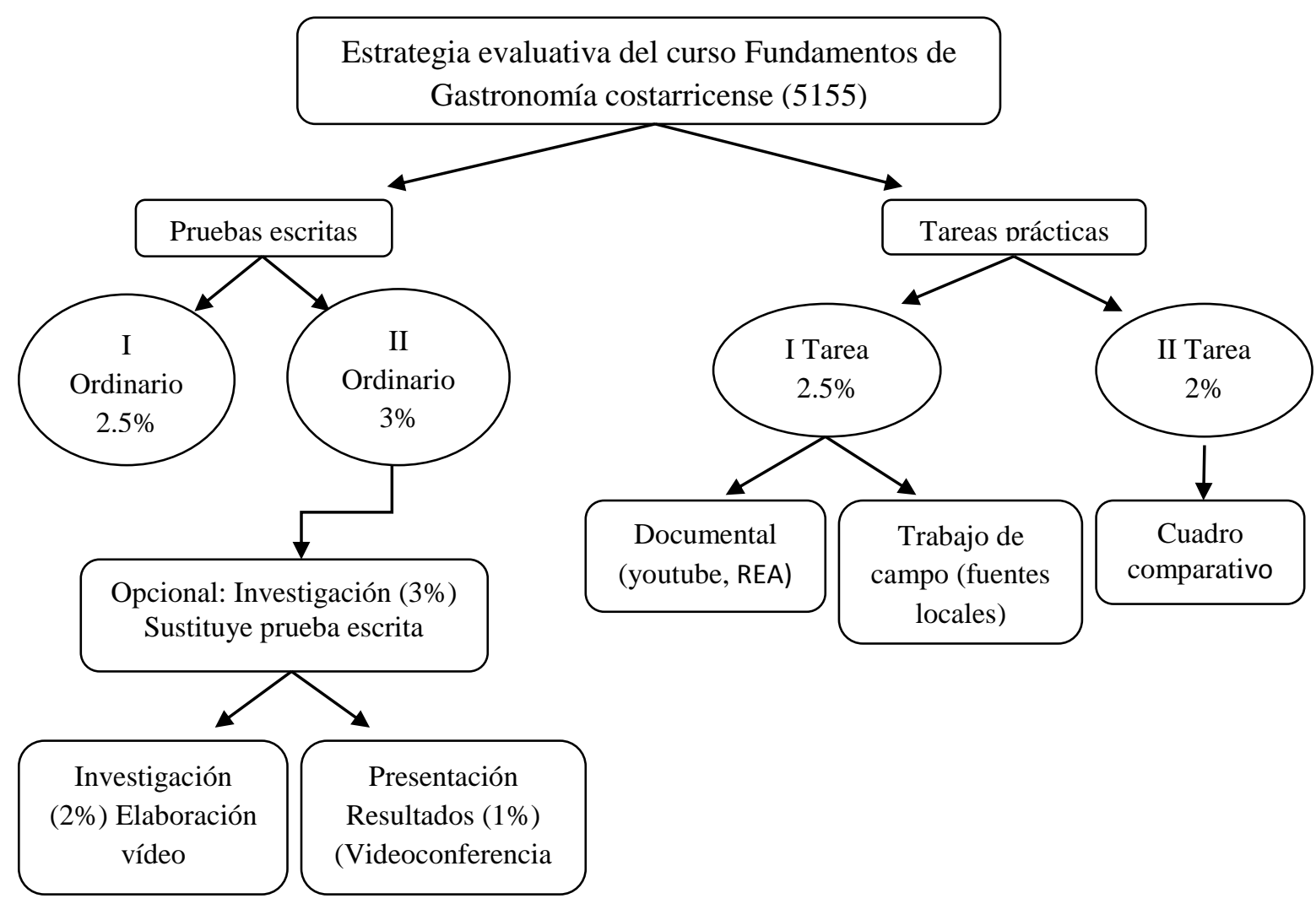

Fuente: Elaboración propia a partir del documento orientación académica (Solís, 2014)

La estrategia evaluativa se estableció visualizando los diversos estilos de aprendizaje es decir, las diversas formas en que una persona aprende (Aragón y Jiménez, 2009), a fin de otorgar opciones que se adapten a las condiciones o a las situaciones particulares de la persona estudiante.

Por un lado, en un primer estadio, las dos pruebas escritas tienen un porcentaje de $5,5 \%$. Sin embargo, hay una leve diferencia con el $4,5 \%$ equivalente a las tareas prácticas. Por lo tanto, se decide dar un porcentaje atractivo a este último rubro, con el objetivo de motivar a los estudiantes a realizar las tareas, para que repasen los contenidos y el desarrollo habilidades específicas; a saber:

En el caso de la primera tarea, al observar el documental dirigido por preguntas generadoras, deberán desarrollar la reflexión y el análisis sobre realidades en un contexto internacional, desde la perspectiva de la globalización. Luego, en la 
segunda sección, deberán desarrollar trabajo de campo para ejecutar la acción de contrastar la teoría con la práctica en su entorno más próximo, su comunidad, pasando de lo global a lo local. Y en la segunda tarea, se vuelve a repasar la acción del contraste utilizando la teoría, pero con la elaboración de un cuadro comparativo.

Por otro lado, en un segundo estadio, se encuentra la opción de desarrollar un proyecto de investigación, el cual sustituye a la prueba escrita para motivar a la persona estudiante a generar sus propios materiales o producciones (creación de video con herramientas gratuitas), con base en los resultados que arrojen sus investigaciones en su comunidad. Antes de presentar su producción, deberá preparar la exposición de resultados (directamente del tema de investigación como de sus lecciones aprendidas en el proceso) que socializará, a través del medio de videoconferencias, con el objetivo de lograr una mejor comunicación y que la persona estudiante se habitúe en la utilización de estos medios de comunicación como futuro profesional.

Las tareas se planificaron para enseñarle a la persona estudiante a aplicar diversos procedimientos, así como reflexiones que, si selecciona la investigación, tiene contextualización e insumo necesarios. En caso de que decida hacer la prueba escrita de igual forma, se asegura que aplicarán las habilidades anteriormente mencionadas.

Con la intención de que la persona estudiante interiorice la autoevaluación para fortalecer su autorregulación, se define tres listas de cotejo o matrices (ver enlace de orientación académica) que orientarán la concreción de las tareas asignadas. De esta forma, se puede estructurar los procesos por seguir, sin apartar los espacios para que la persona estudiante construya su aprendizaje de forma creativa.

En seguida, se presentará el desglose de al menos los primeros instrumentos de evaluación y las actividades desarrolladas al cierre del análisis para la construcción de los datos para la presente ponencia. 
Tabla 2 Desglose de no. de estudiantes por rango de notas obtenidas en actividades de evaluación

\begin{tabular}{|c|c|c|c|c|c|c|c|c|c|}
\hline & \multicolumn{3}{|c|}{ I ordinario } & \multicolumn{3}{|c|}{ I tarea } & \multicolumn{3}{|c|}{ II tarea } \\
\hline & \multicolumn{2}{|c|}{ Estudiantes } & \multirow[t]{2}{*}{$\%$} & \multicolumn{2}{|c|}{ Estudiantes } & \multirow[t]{2}{*}{$\%$} & \multicolumn{2}{|c|}{ Estudiantes } & \multirow[t]{2}{*}{$\%$} \\
\hline $\begin{array}{l}\text { Ubicación } \\
\text { Geográfica }\end{array}$ & Urbano & Rural & & Urbano & Rural & & Urbano & Rural & \\
\hline Rango & & & & & & & & & \\
\hline $100-90$ & 21 & 6 & 28,4 & 29 & 31 & 63 & 30 & 29 & 62 \\
\hline $89-80$ & 11 & 18 & 30,5 & 8 & 13 & 22 & 6 & 11 & 17,9 \\
\hline $79-70$ & 1 & 12 & 13,7 & - & 5 & 5,3 & - & 2 & 2 \\
\hline $\begin{array}{l}\text { Menos de } \\
70\end{array}$ & - & 13 & 13,7 & - & 3 & 3 & - & 5 & 5,2 \\
\hline NSP & 8 & 5 & 13,7 & 4 & 2 & 6,3 & 5 & 7 & 12,7 \\
\hline Total & 41 & 54 & 100 & 41 & 54 & 100 & 41 & 54 & 100 \\
\hline
\end{tabular}

Fuente: Elaboración propia a partir de archivo de notas respectivo (UNED 2014).

Según la tabla anterior, se puede observar una baja deserción o reporte de notas inferiores a $70 \quad(27,4 \%$, en contraposición a $73 \%$ de estudiantes que obtuvieron notas entre los rangos de 100 a 70). Este dato es tipificado como positivo, ya que se muestra una motivación por parte de la persona estudiante a participar de la estrategia evaluativa en sus diferentes etapas en cuenta la II tarea, en la que es usual una alta deserción o bajas calificaciones.

Cuantitativamente, queda demostrado que se cumplió con el objetivo de aprendizaje y, correlacionado cualitativamente, se respalda el resultado. Esto porque, al analizar el desarrollo de los trabajos (tareas), se observa dominio del contenido temático, haciendo un importante contraste entre la teoría y la práctica.

No obstante, se observa una baja y casi nula exposición directa o aporte de la persona estudiante cuando se induce a procesos metacognitivos o a reflexión sobre 
su aprendizaje (actividad lecciones aprendidas); por lo tanto, se deberá ajustar la estrategia para su abordaje.

Si bien es cierto el porcentaje de participación continua y de calidad mostrada, cualitativa y cuantitativamente, detona la necesidad de mejorar los procesos de aprendizaje de la persona estudiante ubicada geográficamente fuera del área metropolitana. A pesar del conocimiento previo que se tiene sobre las grandes brechas socioeconómicas de estas regiones, será un reto afrontar esa meta aunado al hecho de estar ante un modelo educativo a distancia, en el cual muchas veces a la persona estudiante se le dificulta el sistema, pero debe mantenerse porque es la única posibilidad de estudio en su región.

Con respecto al proyecto de investigación, 6 estudiantes seleccionaron la opción y 4 realizaron la exposición de los resultados, a través de videoconferencias. Así que devela dos supuestos, según reflexión de la persona estudiante: el temor a pensar que el proceso de investigación es complejo con un mayor esfuerzo para su realización y que, al desarrollar las dos tareas, contaba con un buen porcentaje en su promedio y que con el mínimo de nota puede ganar el curso.

Otra reflexión que se hace sobre este escenario es que el tiempo y el esfuerzo al ejecutar las dos tareas podría consumirlo para desarrollar una investigación, aunque fuera muy básica. Si del todo no se tiene la cultura de investigar, se hace complejo. La situación es diferente cuando, en otros cursos, se ha sustituido la prueba por un taller y la respuesta de selección, por parte de la persona estudiante, es mucho mayor, aunque de igual forma la limitación, en este caso, será de tiempo y de desplazamiento geográfico.

De una u otra forma, se reforzó el hábito de indagación y de observación Lugo 2005, ya que las tareas prácticas guiaban la aplicación a procesos básicos de investigación, en los cuales es fundamental para su preparación profesional y como persona integral. 
Otro elemento por destacar, al ubicarnos en un contexto de educación a distancia, es que, al establecer la presente estrategia evaluativa, complejiza el proceso de enseñanza y aprendizaje. Por parte de la persona estudiante, debe realizar un esfuerzo para aplicar conocimientos conceptuales, procedimentales y actitudinales, aunados a la utilización de diversos medios. Y la persona docente deberá acompañar y facilitar ese proceso en una matrícula de 95 estudiantes cuando los tiene en un mismo espacio y tiempo.

Ante esta situación, resulta estratégico y necesario elaborar un documento con orientaciones académicas e instrumentos de autorregulación, tal es el caso de las listas de cotejo o, en su efecto, matrices que indican los aspectos que se esperan desarrolle la persona estudiante, para optimizar su aprendizaje y la evaluación respectiva.

En resumen, según la observación efectuada en la plataforma Moodle; del total de estudiantes, aproximadamente, 10\%, ingresó a hacer sus consultas y estas fueron en un nivel básico que, en su mayoría, buscaba reafirmar lo estipulado en el documento Orientación académica.

Respecto de las videoconferencias, que era el medio utilizado para una comunicación sincrónica entre docente y estudiantes, se obtuvo una baja participación, la cual es un aspecto por mejorar, sobre todo, que en el sistema a distancia es una debilidad que la persona estudiante tenga pocos espacios de intercambio o exposición grupal; por lo tanto, esta habilidad debe potencializarse. Además, la riqueza en el intercambio es el aprendizaje en comunidad.

\section{Conclusiones}


Después de analizar los resultados de la información anterior se concluyó que implementar la evaluación como estrategia de aprendizaje resulta de aprovecho en cuanto a formación y orientación a la persona estudiante.

A su vez se demostró que la estrategia evaluativa propuesta mejoró la baja deserción que presentaba el curso en cuestión.

En cuanto al nivel teórico que aborda el curso, la estrategia evaluativa demuestra que levar la teoría a los contextos más próximos a la realidad del estudiante (comunidad o localidad), sin duda alguna, permite una motivación por parte de la persona estudiante. Este aspecto le permitirá retroalimentar e incentivar su aprendizaje.

Para el logro de dicha motivación, es fundamental clarificar bien lo que se desea obtener de la persona estudiante, al desplazarse al campo o práctica. Asimismo, se debe definir su acción, dejando espacios para su creatividad hecho que, en un sistema a distancia, en el cual se generan procesos conductistas se requiere transferirle al estudiantado la confianza y el ambiente, para que cumpla con los parámetros mínimos solicitados y, a su vez, deje llevarse por su creatividad.

Por una parte, ante una asignatura tan teórica, en la cual los contenidos tienen una alta tendencia a la memorización, con la estrategia evaluativa planteada, se logra armonizar y motivar al estudiantado a alcanzar los objetivos de aprendizaje. Y, resuelve la necesidad que se había detectado en el transcurso de la oferta del curso: generar aprendizajes significativos y procesos reflexivos.

Los materiales y apoyos didácticos están vinculados o armonizados con la estrategia evaluativa propuesta, por cuanto esto genera un ambiente de aprendizaje motivador y de cooperación entre estudiantes y docentes. 
Se detecta la necesidad de reforzar en la persona estudiante, los procesos de metacognición así como también, incentivar al desarrollo de la investigación, ya que se presentó un bajo porcentaje de estudiantes que seleccionaron el proyecto de investigación como una opción de proyecto final.

En cuanto a los instrumentos utilizados para la autorregulación que acompañaron la estrategia evaluativa, se demuestra la necesidad e importancia para la vital función del desarrollo del aprender a aprender y para homologar o entender el gran concepto de lo que se desea por parte de la estrategia evaluativa ante la persona estudiante.

Se promovió el uso de la TIC y de los recursos educativos abiertos como fuentes importantes donde la persona estudiante podía obtener información, además de ser un recurso para la expresión de la creatividad Lugo (2005).

Finalmente, la atención constante de la acción docente es esencial, entendida esta como facilitadora o mediadora. A pesar de que los materiales y apoyos didácticos tienen un componente importante de mediación pedagógica, la figura docente deberá orientar, apoyar y motivar al estudiantado, según lo requiera. Este es un esfuerzo mayor, al establecer estrategias evaluativas que generen reto a la persona estudiante y, por supuesto, a la labor docente que deberá afilar su perfil de investigador para develar supuestos que enriquecerán su quehacer.

\section{Capitalización}

La estrategia evaluativa expuesta estableció, según necesidad particular identificada para una asignatura y modelo pedagógico específico (a distancia); no obstante, las dinámicas y las reacciones de los diversos actores involucrados en el proceso de evaluación son muchas veces similares. Por lo tanto, es posible aplicar algunas de esas acciones o propuestas en el tanto se generen cambios en aquello que cada quién considere según contexto. 
En el desarrollo de la experiencia, se incorporan vínculos de internet (enlaces) de referencia, donde la persona interesada pueda consultar los materiales y apoyos didácticos con más detalle; y si tiene a bien, podría utilizar algún instrumento, estrategia o material según su necesidad particular.

Ante un enfoque de evaluación alternativa, construida a partir del uso de diversos medios, recursos y apoyos didácticos, se recomienda generar instrumentos de orientación con alta mediación pedagógica, para no saturar al estudiante ni generar frustración o estrés ante los diversos procesos que deberá desarrollar, aunado el hecho de interactuar con diferentes medios o herramientas digitales y demás. En contraste con lo anterior, es vital mantener un equilibrio entre el tiempo que le puede tomar al estudiante el manejo de un medio de comunicación específico y el estudio o desarrollo del contenido; para ello, se recomienda establecer varios escenarios y opciones, según curva del aprendizaje que se le presente en el grupo.

\section{Referencias}

Álvarez, J. M. (2001). Evaluar para conocer, examinar para excluir, "Página de Inicio". Recuperado de http://unter.org.ar/imagenes/9986.pdf

Aragón G. y Jiménez, Y. (2009). Diagnóstico de los estilos de aprendizaje en los estudiantes: Estrategia docente para elevar la calidad educativa. Recuperado de: http://www.redalyc.org/pdf/2831/283121714002.pdf

Barberà, E. (2004). La educación en la red. Actividades virtuales de la enseñanza y aprendizaje, pp 37-146 Barcelona: Ediciones Paidòs Ibérica, S.A.

Batista, G., Borges, F. y Forès, A. (2008). Didáctica universitaria en Entornos Virtuales, (2 ed.). Madrid: NARCEA, S.A.

Calderón, K. (2008). La vida que enseña. Nociones de Educación Permanente. San José: EUNED.

Carmiol, V. (2010). Aproximación del concepto de autoevaluación de los aprendizajes en el marco del Modelo Pedagógico de la UNED-Costa Rica" "Página de Inicio", Recuperado de file:///G:/Materiales/Mesa_evaluacion_aprendizajes/publicaciones/publicacion_ 7.pdf 
Lugo, A. (2005). Competencia para usar recursos educativos abiertos para la práctica educativa [objeto de aprendizaje], p 2 "Página de Inicio". Recuperado de http://www.ruv.itesm.mx/convenio/tabasco/oas/ureape/homedoc.htm

Solís, S. (2014) Orientaciones académicas asignatura: Fundamentos de Gastronomía Costarricense, Recuperado de http://sanpedro.uned.ac.cr/orientaciones/2014505155.pdf

Universidad Estatal a Distancia (2005). Creación y Organización de la UNED. San José: EUNED.

Universidad Estatal a Distancia (2013). Plan de desarrollo académico UNED 20122017 Recuperado de .http://www.uned.ac.cr/academica/plan_academico/galeria_1.html

Universidad Estatal a Distancia (2014). Archivo de notas asignatura Fundamentos de Gastronomía Costarricense.

Universidad Estatal a Distancia (s.f). Glosario de términos curriculares para la Universidad Estatal a Distancia. Programa de Apoyo Curricular y Evaluación de los Aprendizajes. Recuperado de: http://www.uned.ac.cr/academica/images/PACE/publicaciones/FINAL24-913_Glosario_de_trminos_curriculares_UNED.pdf 